\title{
THE CLUSTER SOFT EXCESS: NEW FACES OF AN OLD ENIGMA
}

\author{
R. LIEU \& J. P. D. MITTAZ \\ Physics Department, $U A H$ \\ Huntsville \\ AL 35899, USA \\ E-mail: lieur@cspar.uah.edu
}

\begin{abstract}
Until the advent of XMM-Newton, the cluster soft excess (CSE) was the subject of some controversy due to both data analysis issues and uncertainties with the soft excess emission mechanism. XMM-Newton observations have finally laid to rest any doubts as to the existence of the CSE and have also given tantalising clues as to the nature of its emission mechanism. Here we report on the analysis of XMMNewton observations of a number of CSE clusters in an attempt to improve the analysis and understanding of the CSE. Included as part of the study is an analysis of the effects of background subtraction, which calls to question the integrity of the claimed O VII line discovery, though not the soft excess itself. We also give details of both thermal and non-thermal fits to the CSE cluster Abell 3112.
\end{abstract}

\section{Introduction}

The cluster soft excess is an excess of flux seen above the hot ICM in a number of clusters first discovered by Lieu et al. (1996), but its origin still remains a mystery. Currently there are two prevalent models, one thermal, normally assumed to arise from warm $(\sim 0.2 \mathrm{keV})$ gas, and one non-thermal. Observations with XMM-Newton have the potential to provide vital new information to resolve this issue and recently there have been claims of Oxygen lines associated with the CSE (Kaastra et al. 2003, Finoguenov, Briel \& Henry 2003). If true this is very important to our understanding of the CSE since it implies that its origin must be thermal rather than nonthermal. However, there are several issues that first need to be addressed before we can be sure that the emission is indeed thermal. The most important is that of background subtraction - to date most CSE studies have used the background dataset of Lumb et al. (2002) exclusively and therefore have not taken into account Galactic background variations across the sky. As we will show, this can cause an overestimate of the CSE as well as 
the spurious detection of $\mathrm{O}$ VII.

\section{Using in-situ backgrounds}

To investigate if variations in the Galactic background are important to the analysis of XMM-Newton data on the CSE we must determine in-situ backgrounds i.e. we must use observations which contain uncontaminated background signal. This will either be when the clusters fit inside the XMM-Newton field of view, or offset pointing which contain both cluster and background flux, Of all the CSE clusters currently studied with XMMNewton to data, only four fit approximately within the XMM-Newton field of view and we discuss two of these, Abell S1101 and Abell 3112. These two clusters are amongst the most important CSE clusters: Abell S1101 has the largest reported ROSAT PSPC soft excess (Bonamente et al. 2001) and Abell 3112 has one of the largest XMM-Newton soft excesses (Nevalainen et al. 2003). For both clusters the estimated contamination of the background by residual cluster emission in the 12'-14' annulus used to estimate the background is small. For these two cluster we can therefore use a double subtraction method (see for example Arnaud et al. 2002) to remove the correct background.

From the point of view of previous analyses of these clusters it is important to compare the different background subtraction techniques. We have therefore compared the Lumb et al. (2002) background used in, for example, Kaastra et al. (2003,2004) (lowest spectrum) with the double subtraction background method using an in-situ background (middle spectrum). Figure 1 shows that there is considerable difference between the Lumb background and the double subtraction background particularly in the case of Abell S1101. Using the Lumb et al. background alone can give rise to a spurious soft X-ray signal which could be confused with a soft excess. However, the model fit to the double background subtracted spectrum (shown as a solid line on the top spectra in Figure 1 together with the fit residuals show that there is still evidence for a soft excess. Therefore, even taking into account the correct Galactic background subtraction, the soft excess still persists.

\section{The $\mathrm{O}$ VII/O VII line region}

One of the key findings of the Kaastra et al. (2003,2004) works was the apparent detection of $\mathrm{O}$ VII in the outer parts of a number of clusters including Abell S1101. The line detection was seen at the outer regions of 

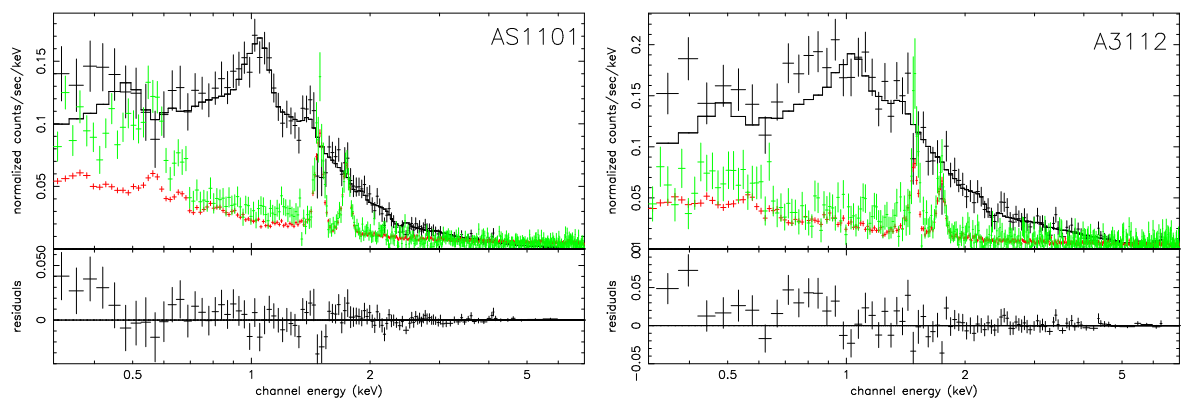

Figure 1. The top spectrum shows the background subtracted spectra from EPIC MOS1 for the 4'-6' arcminute annulus for AS1101 and A3112. The other spectra show the Lumb background (lowest spectrum) and the double subtracted background (middle spectrum). All backgrounds have been normalised to the $10-12 \mathrm{keV}$ source flux. Note the strong $\mathrm{O}$ VIIline seen in the AS1101 background spectra. The solid line is a model for the hot ICM fitted to the $1-7 \mathrm{keV}$ band of the background subtracted spectrum.

clusters and Kaastra et al. directly associated the emission with the CSE. They also related this emission with large extended soft X-ray emission seen in ROSAT all sky survey maps, directly linking the emission with the supercluster environment and hence to the Warm Hot Intergalactic Medium (WHIM). However, in our analysis we find no such line, although the background spectrum of the Abell S1101 field (12'-14') shows strong O VII emission. At first sight, the detection of this line seems to verify the claims of Kaastra et al. but this can be directly tested as the O VII is strong enough to be resolved and we can therefore determine the redshift of the line directly.

The left panel of Figure 2 shows the O VII/O VIII line region together with a model including a continuum model with two gaussians placed at the redshifted energy of the $\mathrm{O}$ VII/O VIII lines. It is clear that putting the Oxygen lines at the redshift of the cluster does not match the observed location of the lines. The right hand panel of Figure 2 shows the case where the lines have been put at zero (Galactic) redshift and it is clear that the lines are much more consistent with a Galactic redshift rather than the cluster redshift. Indeed, if we fit the observed line energies we get $0.569 \pm 0.003 \mathrm{keV}$ for $\mathrm{O}$ VII and $0.651 \pm 0.007 \mathrm{keV}$ for O VIII, completely consistent with zero (Galactic) emission and completely inconsistent with the redshifted line energies $0.538 \mathrm{keV}$ and $0.618 \mathrm{keV}$. We must therefore conclude that the line emission and hence the majority of the emission at the 12 '-14' annulus is Galactic in origin and therefore not associated with the cluster. This then not only verifies our use of the double subtraction method 

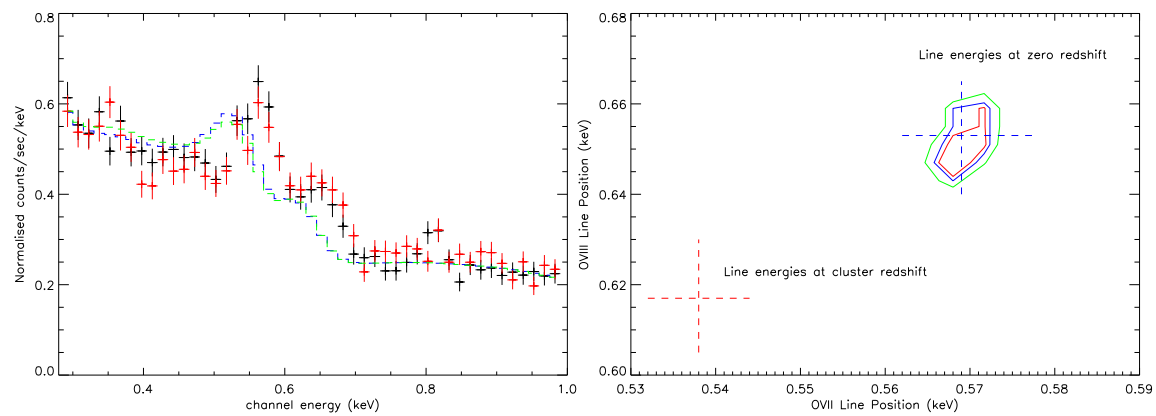

Figure 2. The left panel shows the O VII/O VIIIline region of Abell S1101 showing the two MOS instruments (black $=$ MOS1, red $=$ MOS2), together with models including gaussians placed at the redshift of Abell S1101 ( $\mathrm{z}=0.058)$. It is obvious that the redshifed Oxygen lines do not represent the observed Oxygen lines. The right panel shows the uncertainties to the energies of the O VII and O VIII lines based on fits to the XMM-Newton data. The lines are clearly at the Galactic (zero) redshift rather than the redshift of the cluster

since it shows that the background annulus is dominated by background emission, but calls doubt on the claims of Kaastra et al. $(2003,2004)$ of the association of the CSE with an extended supercluster WHIM emission component.

\section{A case study: Abell 3112}

In order to investigate the impact of our background subtraction method on the observed properties of the soft excess, we have first looked at the cluster with the strongest XMM-Newton CSE, A3112. This cluster was previously studied by Nevalainen et al. (2003) using the Lumb data as the sole background estimator. Figure 1 shows that the Lumb background is not too dissimilar to double subtraction background with the double subtraction background being 30-40\% higher.

Figure 3 shows a broad band (0.3-7 keV) single tempertature fit to all three EPIC instruments to the 1'-2' arcminute annulus. Note that we have only fitted the $0.5-7 \mathrm{keV}$ band for the PN due to calibration problems below $0.5 \mathrm{keV}$ (Kirsch et al. 2004). This single temperature fit is very poor with strong residuals at low energies and a bad $\chi^{2}=1.52$. These are all characteristics of the presence of a strong soft excess in this cluster. We have therefore fitted extra models to the spectrum, on representing a thermal soft excess, one representing a non-thermal model. The fits are shown in Figure 4 with the $\chi^{2}$ values for these fits are $\chi_{\nu}^{2}=1.03$ 
(null hypothesis $=0.28$ ) for the thermal soft excess and $\chi_{\nu}^{2}=1.05$ (null hypothesis $=0.16$ ) for the non-thermal model respectively. Both models have improved significantly over a single temperature fit. However, it is not possible to distinguish between a thermal or non-thermal model. Hopefully, a more detailed analysis of this and other CSE clusters with XMM-Newton will be able to resolve the true emission mechanism.

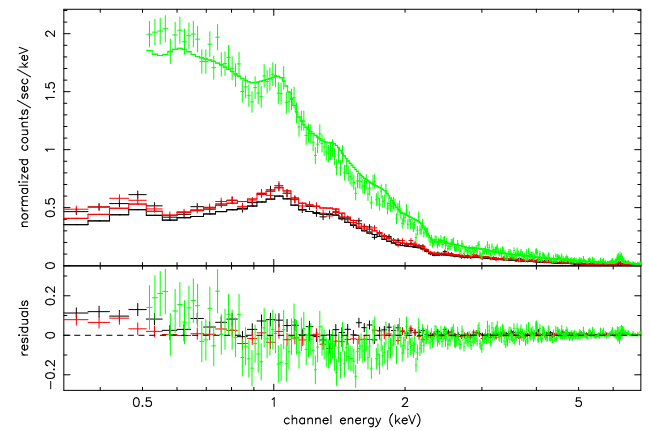

Figure 3. Single temperature broad band fit $(0.3-7 \mathrm{keV})$ to the 1'-2' arcminute annulus for all three EPIC cameras for Abell 3112. Clear low energy residuals and a very poor fit $\left(\chi_{\nu}^{2}=1.52\right.$ null hypothesis $\left.=10^{-16}\right)$ show the presence of a strong soft excess.
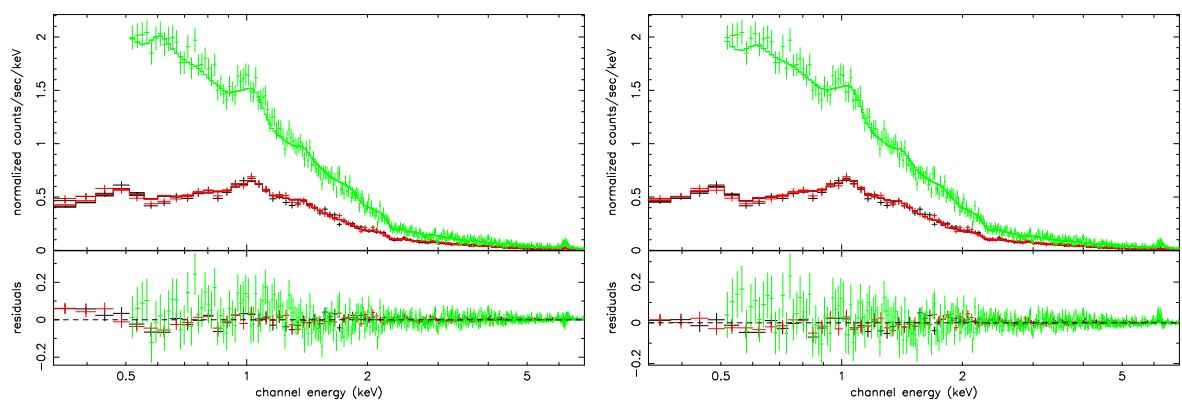

Figure 4. Left panel shows a two temperature model fit to the data (a thermal soft excess) and the right panel shows a hot ICM plus power-law (non-thermal) fit to the same data. The $\chi^{2}$ values for these fits are $\chi_{\nu}^{2}=1.03$ (null hypothesis $=0.28$ ) for the thermal soft excess and $\chi_{\nu}^{2}=1.05$ (null hypothesis $=0.16$ ) for the non-thermal model. Both models have improved significantly over a single temperature fit. 


\section{Discussion}

We have re-assessed the XMM-Newton data analysis of two clusters with reported soft excesses. We find that using a separate background dataset without taking into account the variation of the soft X-ray Galactic background by using in-situ background measurements can lead to an underestimate of the true background and therefore an overestimate of the cluster soft excess. It can also lead to the spurious detection of Galactic $\mathrm{O}$ VII lines which in the past have been ascribed to the clusters themselves. This calls into question many of the conclusions reported in Kaastra et al. $(2003,2004)$. We do find, however, that with the correct background subtraction the cluster soft excesses still exists even though no O VII lines can be seen. Fundamentally, this demonstrates that if we are to correctly understand and parameterise the CSE using XMM-Newton data, the correct background subtraction is absolutely vital. By using the correct background subtraction we then find that instead of having a clear cut emission model as has been recently proposed, we are back to the situation where it is not clear what mechanism gives rise to the CSE.

We have also assessed the physicallity of the different models. With regard to the status of thermal emission from the CSE we cannot completely discount it, but there is no definitive evidence supporting it. On the nonthermal side, emission at the centre may require a non-thermal explanation, but on the outskirts it is not clear if there can be enough energy inputted into the relativistic $\mathrm{CR}$ population to give rise to the strength of the CSE.

Hopefully, new observations and new instruments such as Astro-E2 will be able to finally resolve this issue.

\section{References}

1. Arnaud, M., Neumann, D., Aghanim, N., Gastaud, R., Majerowicz, S., Hughes, J., 2001, A\&A, 365, L80

2. Bonamente, M., Lieu, R. \& Mittaz, J., 2001, ApJ, 561, L63

3. Kaastra, J.S., Lieu, R., Tamura, T., Paerels, F.B.S., den Herder, J.W., 2003, A \& A, 397, 445

4. Kaastra, J.S., Lieu, R., Tamura, T., Paerels, F.B.S., den Herder, J.W., 2004, in 'Soft X-ray emission from clusters of galaxies and related phenomena', eds. R. Lieu \& J, Mittaz (Kluwer Dordrecht), p37

5. Kirsch, M.G.F., Altieri, B., Chen, B., Haberl, F., Metcalfe, L., Pollock, A.M.T., Read, A.M., Saxton, R.D., Sembay, S., Smith, M.J.S., 2004, astro$\mathrm{ph} / 0407257$

6. Lieu, R., Mittaz, J.P.D. et al., 1996, Ap,J., 458, L5

7. Lumb, D.H., Warwick, R.S., Page, M., DeLuca, A., 2002, A\&A, 389, 93 
8. Nevalainen, J., Lieu, R., Bonamente, M., \& Lumb, D. 2003, ApJ, 584, 716 